\title{
Correção das aberrações oculares nos retratamentos de LASIK personalizado e convencional
}

\author{
Correction of ocular aberrations in custom and standard LASIK retreatments
}

\author{
Andréia Peltier Urbano ${ }^{1}$ \\ Walton Nosée
}

Trabalho realizado no Departamento de Oftalmologia da Universidade Federal de São Paulo - UNIFESP- São Paulo (SP) - Brasil.

${ }^{1}$ Doutora em Medicina pelo Curso de Pós-Graduação em Oftalmologia e Ciências Visuais da Universidade Federal de São Paulo - UNIFESP - São Paulo (SP) - Brasil. ${ }^{2}$ Livre-Docente do Departamento de Oftalmologia da UNIFESP - São Paulo (SP) - Brasil; Professor Titular do Departamento de Oftalmologia da Universidade Metropolitana de Santos - UNIMES - Santos (SP) - Brasil.

Endereço para correspondência: Andréia Peltier Urbano. Av. Cardeal da Silva, 2.070/201B - Salvador (BA) CEP 40231-250

E-mail: andreiaurbano@terra.com.br

Recebido para publicação em 21.08.2007

Última versão recebida em 21.09.2009

Aprovação em 04.10.2009

Nota Editorial: Depois de concluída a análise do artigo sob sigilo editorial e com a anuência da Dra. Belquiz Rodrigues A. Nassaralla sobre a divulgação de seu nome como revisora, agradecemos sua participação neste processo.

\section{RESUMO}

Objetivo: Comparar a correção das aberrações oculares nos retratamentos de LASIK personalizado e convencional. Métodos: Foi realizado um estudo prospectivo, randomizado, de 74 olhos de 37 pacientes submetidos ao retratamento de LASIK para correção de miopia e astigmatismo. Cada paciente foi submetido ao retratamento de LASIK personalizado Zyoptix (Bausch \& Lomb) em um olho e convencional Planoscan (Bausch \& Lomb) no olho contralateral. Foi comparada a correção das aberrações oculares nos retratamentos personalizado e convencional. Resultados: No sexto mês pós-operatório, os olhos submetidos ao retratamento Zyoptix apresentaram diminuição estatisticamente significativa do defocus, astigmatismo, coma, aberração esférica, segunda ordem, terceira ordem, alta ordem e aberrações totais. Os olhos submetidos ao retratamento Planoscan apresentaram diminuição estatisticamente significativa do defocus, segunda ordem e aberrações totais. Conclusões: $\mathrm{O}$ retratamento personalizado foi superior ao retratamento convencional para a correção das aberrações oculares de baixa e alta ordens.

Descritores: Astigmatismo/cirurgia; Astigmatismo/fisiopatologia; Miopia/cirurgia; Miopia/ fisiopatologia; Ceratomileuse assistida por excimer laser in situ/métodos; Acuidade visual/ fisiologia; Retratamento

\section{INTRODUÇÃO}

O uso da análise de frentes de ondas na Oftalmologia proporcionou um grande avanço diagnóstico e terapêutico no campo da Cirurgia Refrativa. Com esta tecnologia, tornou-se possível mensurar as aberrações de baixa e alta ordens do sistema óptico. Do ponto de vista prático, as aberrações de baixa ou segunda ordem - o defocus e o astigmatismo, que equivalem aos componentes esfero-cilíndrico da refração clínica - podem ser corrigidas, clinicamente (através do uso de óculos ou lentes de contato) ou cirurgicamente (com a ablação convencional ou personalizada). As aberrações de alta ordem, no entanto, podem ser corrigidas ou minimizadas, cirurgicamente, com a ablação personalizada ${ }^{(1)}$.

Atualmente, sabe-se que a cirurgia refrativa convencional baseada na fórmula de Munnerlyn tende a induzir aberrações oculares de alta ordem enquanto corrige as aberrações oculares de baixa ordem. $\mathrm{O}$ aumento destas aberrações de alta ordem correlaciona-se com as queixas de qualidade visual após a cirurgia refrativa convencional (sintomas de ofuscamento e halos noturnos) $)^{(2-3)}$.

A cirurgia refrativa personalizada baseada na análise de frentes de ondas objetiva corrigir ou minimizar as aberrações oculares de alta ordem 
enquanto corrige as aberrações de baixa ordem. Desta maneira, ao proporcionar uma superfície ocular pós-operatória mais homogênea, oferece uma melhor qualidade visual pósoperatória ${ }^{(4)}$.

Os resultados das cirurgias refrativas primárias personalizadas têm sido promissores quando comparados aos resultados das cirurgias refrativas primárias convencionais ${ }^{(5-6)}$. Optamos por realizar um estudo comparativo da correção das aberrações oculares em casos de retratamento de LASIK convencional, com as técnicas de ablação personalizada e convencional.

\section{OBJETIVO}

Comparar a correção das aberrações oculares em casos de retratamento de LASIK primário convencional com as técnicas de ablação personalizada e ablação convencional.

\section{MÉTODOS}

Foi realizado um estudo prospectivo, randomizado, de 74 olhos de 37 pacientes submetidos ao retratamento de LASIK para correção de miopia e astigmatismo.

O grupo de estudo foi constituído por um dos olhos de cada paciente submetido ao retratamento pela técnica de LASIK com ablação personalizada Zyoptix (Bausch \& Lomb, Munique, Baviera). O grupo-controle foi composto pelo olho contralateral submetido ao retratamento pela técnica de LASIK com ablação convencional Planoscan (Bausch \& Lomb, Munique, Baviera). A escolha do olho a ser submetido ao retratamento com a técnica personalizada ou convencional foi aleatória.

Os pacientes realizaram cirurgia de LASIK primária com a técnica convencional, no período de novembro de 1995 a fevereiro de 2003, por um único cirurgião, na Eye Clinic Day Hospital (São Paulo, Brasil). Os retratamentos de LASIK foram realizados, no período de agosto de 2002 a outubro de 2003, com levantamento do disco da cirurgia anterior, por um único cirurgião, na Eye Clinic Day Hospital (São Paulo, Brasil). Após a cirurgia de retratamento de LASIK, os pacientes foram acompanhados por um período de seis meses.

Todos os pacientes assinaram consentimento, sendo devidamente informados, após explanação do procedimento, de potenciais benefícios e riscos. O estudo foi avaliado e aprovado pelo Comitê de Ética da Universidade Federal de São Paulo.

Os critérios de inclusão foram: cirurgia de LASIK primária prévia, bilateral e com correção pela técnica convencional; erros refrativos similares antes da cirurgia primária (equivalente esférico inferior a uma dioptria entre os dois olhos); erros refrativos residuais similares antes do retratamento (equivalente esférico inferior a uma dioptria entre os dois olhos) e idade superior a 18 anos.

Os critérios de exclusão foram: distúrbios oculares além de erros refrativos; doenças sistêmicas com manifestações oculares; cirurgias intraoculares prévias (facectomia e vitrecto- mia); ocorrência de mais de uma cirurgia refrativa em cada olho; técnicas cirúrgicas diferentes entre os dois olhos; melhor acuidade visual corrigida inferior a 20/25; diferença de acuidade visual entre os dois olhos superior a uma linha da melhor acuidade visual corrigida e contraindicação cirúrgica para nova cirurgia de LASIK. O retratamento de LASIK foi contraindicado quando o valor calculado presumido para o estroma residual no pós-operatório foi inferior a 250 micrômetros $(\mu \mathrm{m})$ e o valor calculado presumido para a ceratometria corneana pós-operatória inferior a 37 dioptrias (D).

No pré-operatório, os pacientes foram submetidos a exame oftalmológico completo e aberrometria com sensor de frente de ondas Zywave ${ }^{\mathrm{TM}}$ (tipo Shack-Hartmann) - versão 3.21 (Bausch \& Lomb, Munique, Baviera). Para programação cirúrgica, foram analisados os valores da "provável refração do foróptero" (PPR) para pupila de 3,5 $\mathrm{mm}$ em dioptrias (componentes esférico, cilíndrico e eixo) de imagens da aberrometria com diâmetro da pupila superior a $6,0 \mathrm{~mm}$.

Todas as cirurgias de retratamento foram realizadas por um único cirurgião, pela técnica cirúrgica de LASIK personalizado Zyoptix (grupo de estudo) e pela técnica cirúrgica de LASIK convencional Planoscan (grupo-controle). Em todos os pacientes, a ablação do estroma corneal foi realizada após levantamento do disco, com o aparelho de excimer laser Technolas $217 \mathrm{Z}^{\mathrm{TM}}$ (Bausch \& Lomb, Munique, Baviera). Para o período pós-operatório, foram prescritos para todos os pacientes colírios de: tobramicina a $0,3 \%$, cetorolaco de trometamina a $0,5 \%$, acetato de prednisolona a $0,12 \%$ e hipromelose a $0,5 \%$.

As avaliações pós-operatórias foram realizadas após um dia, uma semana e um, três e seis meses. Toda a avaliação pré e pósoperatória foi realizada por um mesmo examinador.

\section{Método estatístico}

A análise estatística foi realizada com o Programa Statistical Package for the Social Sciences SPSS (SPSS Inc., Chicago, Illinois) na versão 10.0 para Windows.

Os valores do RMS das aberrações oculares foram descritos com a média e desvio-padrão para o diâmetro da pupila de $6,0 \mathrm{~mm}$. A comparação entre as médias dos períodos pré-operatório e pós-operatório foi realizada com o teste de Wilcoxon, separadamente, para os grupos Zyoptix e Planoscan.

A comparação das médias dos valores entre os grupos Zyoptix e Planoscan, em cada período avaliado, foi obtida pelo teste de Mann-Whitney.

Para avaliar a modificação das aberrações oculares, foram calculados o porcentual de correção e o fator de correção destas aberrações. Se o valor do porcentual de correção foi negativo, considerou-se que a aberração ocular foi reduzida no pós-operatório. Se o valor do fator de correção foi abaixo de um, considerou-se que a aberração foi reduzida no período pós-operatório.

As médias e desvio-padrão do percentual de correção e do fator de correção entre os grupos Zyoptix e Planoscan foram comparados com o teste de Mann-Whitney no período do sexto mês pós-operatório. 


\section{RESULTADOS}

\section{Comparação das aberrações oculares entre os períodos pré-operatório e pós-operatório, nos grupos Zyoptix e Planoscan}

A análise dos valores das aberrações oculares no préoperatório em relação ao período pós-operatório de seis meses nos grupo Zyoptix e no grupo Planoscan está evidenciada na tabela 1 .

No grupo Zyoptix, houve diminuição estatisticamente significativa do defocus, astigmatismo, coma, aberração esférica, segunda ordem (baixa ordem), terceira ordem, alta ordem e aberrações totais. As aberrações de quinta ordem apresentaram aumento estatisticamente significativo e as demais aberrações não apresentaram diferença significativa entre os períodos pré e pós-operatório de seis meses.

No grupo Planoscan, houve diminuição estatisticamente significativa do defocus, segunda ordem (baixa ordem) e aberrações totais. Houve aumento significativo das aberrações coma secundário e de quinta ordem, sem diferença significativa para as demais aberrações.

\section{Comparação das aberrações oculares entre os grupos Zyoptix e Planoscan}

A comparação dos valores das aberrações oculares entre os grupos Zyoptix e Planoscan dos exames realizados no período pré-operatório do retratamento está apresentada na figura 1. Não houve diferença estatisticamente significativa das aberrações entre os grupos, com exceção do tetrafoil, que, no grupo Zyoptix, teve valor médio de $0,10 \pm 0,07 \mu \mathrm{m}$, e, no grupo Planoscan, teve valor de $0,14 \pm 0,10 \mu \mathrm{m}(\mathrm{p}=0,03)$.
No acompanhamento de seis meses, o grupo Zyoptix apresentou valores estatisticamente inferiores aos do grupo Planoscan das aberrações defocus, coma, trefoil, segunda ordem, terceira ordem, alta ordem e aberrações totais (Figura 2).

\section{Análise do fator e porcentual de correção das aberrações oculares entre os grupos Zyoptix e Planoscan}

Para a comparação dos resultados da correção das aberrações entre os grupos Zyoptix e Planoscan, devemos considerar que o fator de correção com valores abaixo de um e a porcentagem de correção com sinal negativo correspondem à redução da aberração no período pós-operatório (Tabela 2).

No sexto mês, as aberrações defocus, o grupo da segunda ordem (baixa ordem) e o grupo das aberrações totais apresentaram redução no pós-operatório nos dois grupos, sendo que a correção obtida no grupo Zyoptix foi, estatisticamente, mais significativa do que no grupo Planoscan. Os valores das aberrações coma, aberração esférica, do grupo da terceira ordem e do grupo das aberrações de alta ordem foram reduzidos após a cirurgia personalizada Zyoptix, enquanto que, na cirurgia convencional, estes valores apresentaram aumento no pós-operatório, com diferença estatística entre os dois grupos. No grupo Zyoptix, a correção do astigmatismo secundário apresentou um fator de 1,93 $\pm 2,70$, ou seja, $+93,21 \pm 270,2 \%$, enquanto no grupo Planoscan a correção foi com um fator de $1,04 \pm 0,55$, ou seja, $+3,71 \pm 55,36$.

\section{DISCUSSÃO}

Quando analisamos os resultados da correção das aberrações oculares no retratamento de LASIK, podemos observar

\begin{tabular}{|c|c|c|c|c|c|c|}
\hline & \multicolumn{6}{|c|}{ Mudança das aberrações oculares } \\
\hline & \multicolumn{3}{|c|}{ Zyoptix } & \multicolumn{3}{|c|}{ Planoscan } \\
\hline & Pré-op. & Pós-op. (seis meses) & Valor de $p$ & Pré-op. & Pós-op. (seis meses) & Valor de $p$ \\
\hline Segunda ordem & $3,24 \pm 1,44$ & $0,88 \pm 0,69$ & 0,01 & $3,18 \pm 1,09$ & $1,65 \pm 0,84$ & 0,010 \\
\hline Defocus & $3,14 \pm 1,45$ & $0,68 \pm 0,68$ & 0,01 & $3,08 \pm 1,13$ & $1,52 \pm 0,86$ & 0,010 \\
\hline Astigmatismo & $0,61 \pm 0,44$ & $0,45 \pm 0,31$ & 0,03 & $0,64 \pm 0,40$ & $0,54 \pm 0,26$ & 0,250 \\
\hline Terceira ordem & $0,55 \pm 0,27$ & $0,41 \pm 0,18$ & 0,01 & $0,54 \pm 0,29$ & $0,55 \pm 0,24$ & 0,410 \\
\hline Coma & $0,48 \pm 0,26$ & $0,31 \pm 0,18$ & 0,01 & $0,45 \pm 0,29$ & $0,44 \pm 0,25$ & 0,670 \\
\hline Trefoil & $0,24 \pm 0,15$ & $0,23 \pm 0,11$ & 0,91 & $0,26 \pm 0,17$ & $0,29 \pm 0,12$ & 0,015 \\
\hline Quarta ordem & $0,51 \pm 0,27$ & $0,46 \pm 0,18$ & 0,05 & $0,50 \pm 0,19$ & $0,50 \pm 0,21$ & 0,990 \\
\hline Aberração esférica & $0,46 \pm 0,29$ & $0,40 \pm 0,22$ & 0,01 & $0,44 \pm 0,19$ & $0,45 \pm 0,23$ & 0,810 \\
\hline Astigmatismo sec. & $0,13 \pm 0,10$ & $0,14 \pm 0,07$ & 0,22 & $0,14 \pm 0,09$ & $0,12 \pm 0,07$ & 0,310 \\
\hline Tetrafoil & $0,10 \pm 0,07$ & $0,12 \pm 0,06$ & 0,15 & $0,14 \pm 0,10$ & $0,13 \pm 0,06$ & 0,640 \\
\hline Quinta ordem & $0,11 \pm 0,09$ & $0,14 \pm 0,06$ & 0,01 & $0,09 \pm 0,06$ & $0,15 \pm 0,06$ & 0,010 \\
\hline Coma sec. & $0,07 \pm 0,09$ & $0,06 \pm 0,04$ & 0,84 & $0,05 \pm 0,04$ & $0,07 \pm 0,04$ & 0,030 \\
\hline Trefoil sec. & $0,05 \pm 0,03$ & $0,05 \pm 0,03$ & 0,77 & $0,04 \pm 0,03$ & $0,05 \pm 0,02$ & 0,100 \\
\hline Pentafoil & $0,06 \pm 0,04$ & $0,07 \pm 0,04$ & 0,07 & $0,06 \pm 0,05$ & $0,06 \pm 0,04$ & 0,430 \\
\hline Baixa ordem & $3,24 \pm 1,44$ & $0,88 \pm 0,69$ & 0,01 & $3,18 \pm 1,09$ & $1,65 \pm 0,84$ & 0,010 \\
\hline Alta ordem & $0,78 \pm 0,35$ & $0,65 \pm 0,22$ & 0,01 & $0,77 \pm 0,31$ & $0,78 \pm 0,27$ & 0,480 \\
\hline RMS total & $3,35 \pm 1,43$ & $1,15 \pm 0,63$ & 0,01 & $3,29 \pm 1,09$ & $1,85 \pm 0,82$ & 0,010 \\
\hline
\end{tabular}




\begin{tabular}{|c|c|c|c|c|c|}
\hline \multicolumn{6}{|c|}{ Correção das aberrações oculares - seis meses } \\
\hline & \multicolumn{2}{|c|}{ Zyoptix } & \multicolumn{2}{|c|}{ Planoscan } & \multirow[b]{2}{*}{ Valor de $p$} \\
\hline & Fator & $(\%)$ & Fator & $(\%)$ & \\
\hline Segunda Ordem & $0,32 \pm 0,27$ & $-68,24 \pm 27,30$ & $0,59 \pm 0,40$ & $-41,24 \pm 40,06$ & $<0,001$ \\
\hline Defocus & $0,26 \pm 0,28$ & $-74,28 \pm 27,85$ & $0,61 \pm 0,65$ & $-38,70 \pm 65,19$ & $<0,001$ \\
\hline Astigmatismo & $0,98 \pm 0,61$ & $-2,19 \pm 61,36$ & $1,42 \pm 1,52$ & $+41,70 \pm 152,32$ & 0,740 \\
\hline Terceira Ordem & $0,81 \pm 0,40$ & $-19,44 \pm 40,16$ & $1,23 \pm 0,62$ & $+22,94 \pm 62,39$ & $<0,001$ \\
\hline Coma & $0,72 \pm 0,52$ & $-27,66 \pm 51,53$ & $1,15 \pm 0,70$ & $+15,17 \pm 69,82$ & $<0,001$ \\
\hline Trefoil & $1,24 \pm 0,89$ & $+23,61 \pm 89,06$ & $1,52 \pm 1,12$ & $+52,41 \pm 111,63$ & 0,150 \\
\hline Quarta Ordem & $1,03 \pm 0,46$ & $+2,67 \pm 45,56$ & $1,05 \pm 0,28$ & $+4,87 \pm 27,81$ & 0,150 \\
\hline Aberração esférica & $0,85 \pm 0,30$ & $-15,43 \pm 30,16$ & $1,03 \pm 0,32$ & $+2,76 \pm 31,82$ & 0,030 \\
\hline Astigmatismo sec. & $1,93 \pm 2,70$ & $+93,21 \pm 270,25$ & $1,04 \pm 0,55$ & $+3,71 \pm 55,36$ & 0,040 \\
\hline Tetrafoil & $1,94 \pm 2,07$ & $+94,44 \pm 206,76$ & $1,64 \pm 1,68$ & $+64,27 \pm 168,29$ & 0,230 \\
\hline Quinta Ordem & $1,68 \pm 1,00$ & $+68,15 \pm 99,99$ & $2,04 \pm 1,14$ & $+104,03 \pm 114,18$ & 0,270 \\
\hline Coma sec. & $2,12 \pm 2,14$ & $+112,31 \pm 213,70$ & $1,90 \pm 1,59$ & $+89,72 \pm 158,70$ & 0,700 \\
\hline Trefoil sec. & $1,42 \pm 1,13$ & $-73,49 \pm 19,72$ & $3,20 \pm 5,71$ & $-73,10 \pm 27,42$ & 0,250 \\
\hline Pentafoil & $1,39 \pm 0,79$ & $+39,30 \pm 78,92$ & $1,67 \pm 1,38$ & $+66,74 \pm 138,22$ & 0,830 \\
\hline Baixa ordem & $0,32 \pm 0,27$ & $-68,24 \pm 27,30$ & $0,59 \pm 0,40$ & $-41,24 \pm 40,06$ & $<0,001$ \\
\hline Alta ordem & $0,89 \pm 0,26$ & $-11,19 \pm 26,07$ & $1,13 \pm 0,36$ & $+12,78 \pm 35,92$ & $<0,001$ \\
\hline RMS tatal & $0,38 \pm 0$ & $-62,33 \pm 23,00$ & $0,62 \pm 0$ & $-37,70 \pm 34,00$ & $<0,001$ \\
\hline
\end{tabular}

que, no grupo personalizado Zyoptix, houve diminuição significativamente estatística dos componentes defocus, astigmatismo, coma e aberração esférica e dos grupos de segunda e terceira ordem, baixa ordem, alta ordem e total, no sexto mês pós-operatório. Sendo assim, o retratamento personalizado foi efetivo na correção das aberrações de baixa ordem (defocus e astigmatismo) e nas aberrações de alta ordem (especialmente do coma e aberração esférica). Houve aumento significativamente estatístico das aberrações de quinta ordem no período estudado. Podemos observar que a cirurgia personalizada cumpriu o seu objetivo primordial da correção adequada das aberrações de segunda ordem e as de alta ordem, principalmente o coma e aberração esférica. Estas são as principais aberrações de alta ordem que se objetiva corrigir com a ablação personalizada com os sistemas de lasers atuais que utilizam feixes de lasers de 0,8 a 1,0 $\mu \mathrm{m}$. Já foi demonstrado por Huang et al. ${ }^{(7)}$ que a correção das aberrações acima de quarta ordem seria possível com a utilização de feixes de lasers com diâmetros menores. Desta forma, era esperado que as aberrações de quinta ordem aumentassem após a cirurgia personalizada devido à limitação do feixe de laser para a correção destas aberrações de pequena magnitude.

No retratamento convencional Planoscan deste estudo, houve redução estatística da aberração defocus, do grupo da segunda ou baixa ordem e das aberrações totais. Estes resultados apontam para a eficiência deste retratamento na correção das aberrações de baixa ordem. Um resultado interessante a ser observado é que as aberrações de terceira e quarta ordem não apresentaram aumento significativamente estatístico em relação ao pré-operatório. Este resultado é diferente dos encontrados cirurgias primárias personalizadas de LASIK e PRK, onde se pode encontrar aumento das aberrações de terceira e quarta ordem ${ }^{(8-14)}$. Apenas o coma secundário e o grupo de quinta ordem apresentaram aumento significativo em relação ao pré-operatório.

Ao compararmos os valores das aberrações entre os dois grupos, podemos observar que o grupo Zyoptix apresentou médias estatisticamente inferiores para as aberrações defocus, trefoil, coma, de segunda, de terceira, de alta ordem e total no período estudado. Isto sugere que o retratamento de LASIK personalizado foi superior ao retratamento convencional na correção destas aberrações. Embora tenha havido aumento significativo do astigmatismo secundário no grupo Zyoptix isto deve ter ocorrido devido a algum caso isolado fora do padrão ("outlier"), como pode ser observado pelo alto desvio-padrão da média desta aberração (270,2\%), não sendo representativo da amostra global.

Os fatores de modificação das aberrações oculares encontrados neste estudo são comparáveis aos dos estudos publicados sobre LASIK primário personalizado. Devemos ressaltar que, embora estejamos realizando a comparação com outros estudos, esta análise deve ser feita com a ressalva, pois os estudos são heterogêneos quanto à plataforma de tratamento personalizado utilizada, zona óptica do tratamento, médias de equivalentes esféricos da amostra e tamanho da pupila para análise das aberrações. A depender do estudo, o grupo de alta ordem pode ser composto por aberrações até quarta, quinta ou sexta ordem.

De uma forma sucinta podemos observar que, após a cirurgia de LASIK convencional há um aumento significativo das aberrações de alta ordem, especialmente devido ao aumento do coma e da aberração esférica. 


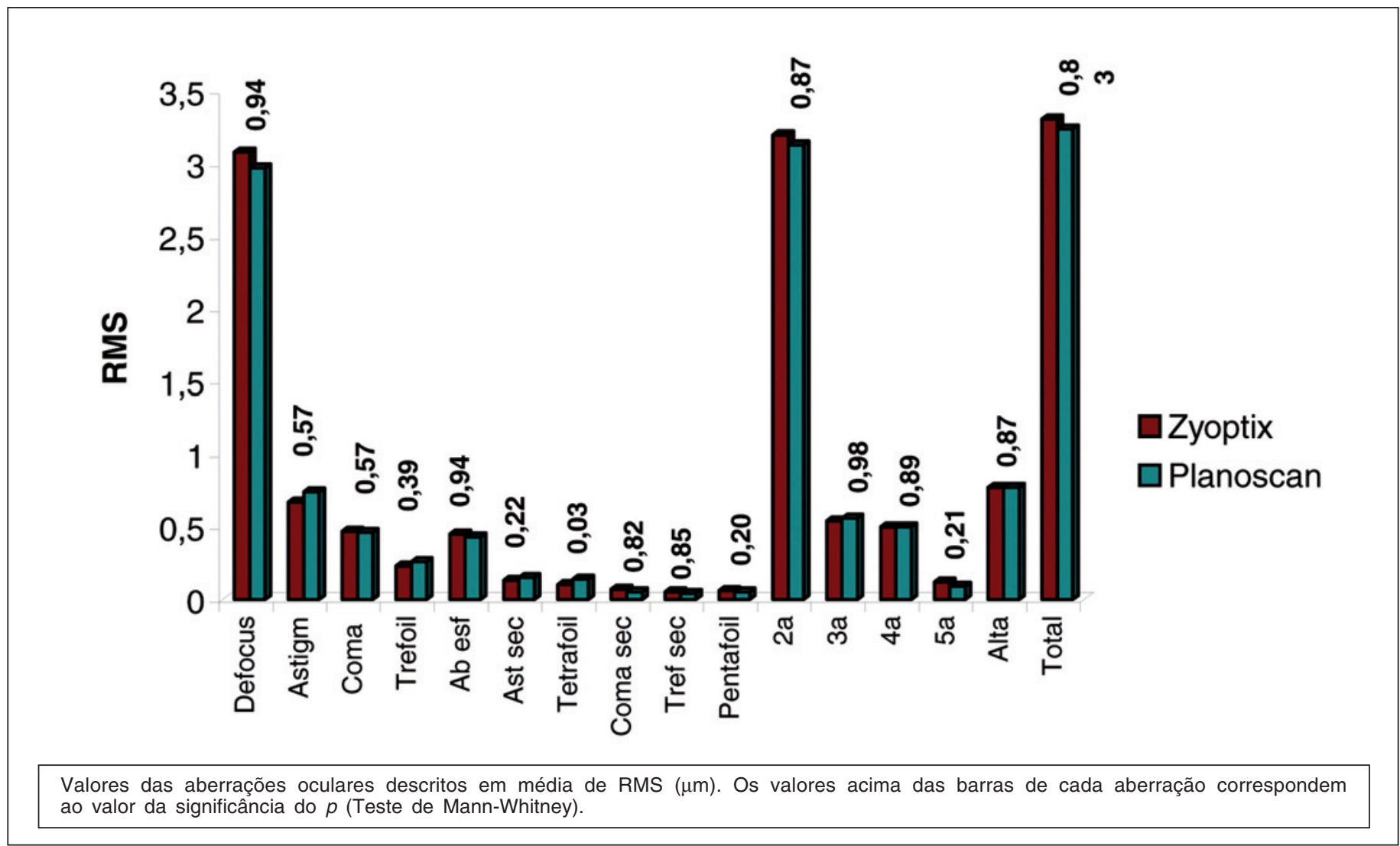

Figura 1 - Comparação das médias do RMS das aberrações oculares entre os grupos Zyoptix e Planoscan no período pré-operatório do retratamento.

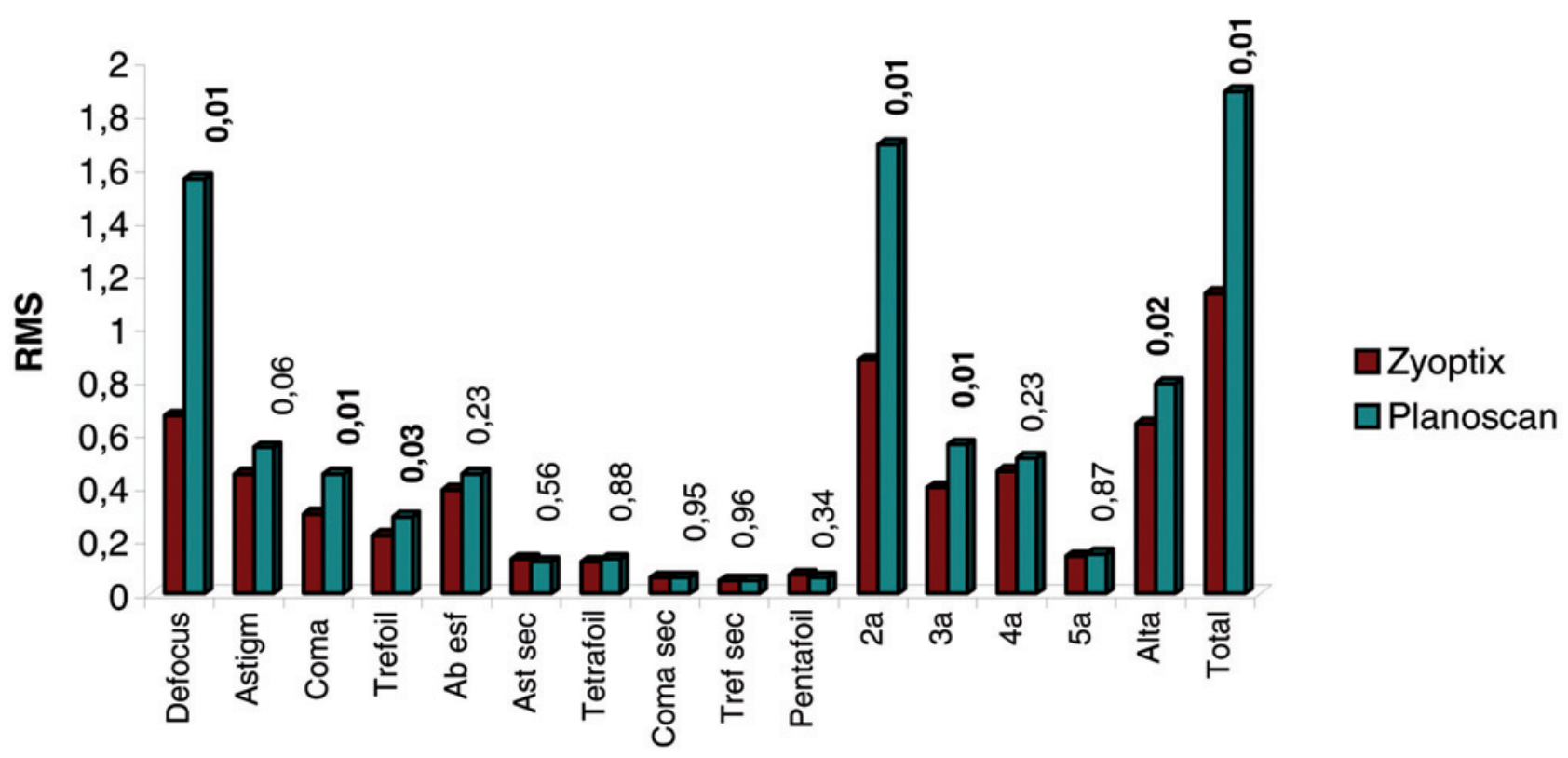

Os valores das aberrações oculares descritos em média de RMS $(\mu \mathrm{m})$. Os valores acima das barras de cada aberração correspondem ao valor da significância do $p$ (Teste de Mann-Whitney).

Figura 2 - Comparação das médias dos RMS das aberrações oculares entre os grupos Zyoptix e Planoscan em seis meses. 
O que observamos após a cirurgia de LASIK primária personalizada é que pode haver uma menor indução das aberrações de alta ordem ou uma pequena redução dos valores. A maior parte dos estudos relata um aumento do valor do grupo da alta ordem, variando com uma porcentagem de aumento entre $2,94 \%{ }^{(15)}$. a $79,22 \%{ }^{(16)}$. No estudo comparativo entre as plataformas CustomCornea (Alcon, Fort Worth, Texas) e CustomVue (VISX Inc., Santa Clara, Califórnia), no entanto, foram observadas diminuição do grupo da alta ordem após cirurgia de LASIK primária personalizada, de $12 \%$ e $12,5 \%$, respectivamente $^{(17)}$. Quando analisamos os termos de Zernike após a cirurgia de LASIK primária personalizada podemos observar que a correção da aberração coma e da aberração esférica variou com o estudo. Alguns autores não evidenciaram modificação da aberração coma após a cirurgia primária personalizada ${ }^{(18)}$, outros detectaram aumento variando entre $23 \%{ }^{(19)}$ a $57,9 \%{ }^{(20)}$, enquanto outros apresentaram redução do coma entre 15,4\% a $33 \%{ }^{(17)}$. A maior parte dos pesquisadores verificou aumento para aberração esférica variando entre $1 \%{ }^{(19)}$ a $431 \%{ }^{(21)}$.

Os resultados comparativos das aberrações oculares após as técnicas de LASIK primária personalizada e convencional sugerem que a indução das aberrações de alta ordem encontrada após a cirurgia personalizada é menor que a indução da cirurgia convencional, embora estas diferenças não sejam estatisticamente diferentes. No estudo de Kim et al. ${ }^{(16)}$, após a cirurgia de LASIK primária personalizada com a plataforma Zyoptix (Bausch \& Lomb, Munique, Baviera), houve um aumento das aberrações de alta ordem de 79,22\%, enquanto após a cirurgia de LASIK convencional com a plataforma Planoscan (Bausch \& Lomb, Munique, Baviera) esta indução foi de $94,4 \%$. No estudo de Vongthongsri et al. ${ }^{(22)}$, houve um aumento das aberrações de alta ordem de $111 \%$ nos olhos submetidos à cirurgia de LASIK primária personalizada com a plataforma Navex (Nidek Co., Gamagori, Japão) e 115\% nos olhos com LASIK primário convencional.

Quando analisamos os resultados da correção das aberrações oculares após o retratamento personalizado na literatura, podemos observar que o retratamento é eficiente na redução das aberrações de alta ordem ${ }^{(4,23-24)}$. As aberrações de alta ordem foram reduzidas, em média, $32,4 \%{ }^{(25)}$ a $72,9 \%{ }^{(26)}$, a aberração coma em $14 \%^{(15)}$ a $72,2 \%{ }^{(26)}$ e a aberração esférica em $28,8 \%{ }^{(26)}$ a $50,8 \%{ }^{(24)}$.

Em nosso estudo, podemos observar que, no acompanhamento de seis meses após a cirurgia de retratamento personalizada Zyoptix, houve uma redução média em 11,19\% do grupo de alta ordem e em 27,66\% do coma e em 15,43\% da aberração esférica. No grupo do retratamento convencional Planoscan, houve um aumento de $12,8 \%$ das aberrações de alta ordem, em $15,17 \%$ do coma e $2,76 \%$ da aberração esférica.

Podemos notar que os resultados das aberrações oculares após o retratamento de LASIK personalizado com o sistema Zyoptix são superiores aos obtidos após a cirurgia de LASIK primária personalizada. Os resultados do retratamento convencional Planoscan são comparáveis e até superiores aos de alguns estudos sobre cirurgias de LASIK primária personalizada ${ }^{(16,21)}$.
Acreditamos que o retratamento personalizado do LASIK neste estudo proporcionou uma melhor correção das aberrações de alta ordem pelos motivos a seguir. Primeiramente, como o retratamento de LASIK foi realizado pela técnica de levantamento do disco e sem a necessidade de confecção de um novo disco, podemos ter evitado a indução de aberrações geradas pelo corte do microceratótomo. Desta forma, as aberrações geradas pela confecção do primeiro disco e pela primeira ablação foram mensuradas antes do retratamento e consideradas para a correção personalizada. Em segundo lugar, atualmente se sabe que a correção das aberrações oculares está relacionada com a sua magnitude pré-operatória ${ }^{(27)}$. Como os olhos que necessitam de retratamento tendem a apresentar mais aberrações de alta ordem que os olhos normais, isto pode explicar porque o retratamento personalizado proporcionou melhor correção destas aberrações.

\section{CONCLUSÕES}

O presente estudo evidenciou que o retratamento personalizado Zyoptix foi superior ao retratamento convencional Planoscan na correção das aberrações oculares de baixa e alta ordens e proporcionou maior estabilidade das aberrações oculares até o sexto mês pós-operatório.

\section{AGRADECIMENTOS}

Agradecimento à Direção, colegas e funcionários da Eye Clinic pelo grande incentivo e importante contribuição neste estudo.

\section{ABSTRACT}

Purpose: To compare the correction of ocular aberrations between custom and standard LASIK retreatment. Methods: Prospective, randomized trial with paired eye control of 74 eyes from 37 patients who underwent LASIK retreatment. Each patient underwent retreatment using Zyoptix LASIK (Bausch \& Lomb) in 1 eye and Planoscan LASIK (Bausch \& Lomb) in the fellow eye. Correction of ocular aberrations was compared between custom and standard LASIK retreatments. Results: At 6 months, there was a statistically significant reduction in defocus, astigmatism, coma, spherical aberration, second, third, higher-order and total aberration in Zyoptix eyes. There was a statistically significant reduction in defocus, second-order and total aberration in Planoscan eyes. Conclusions: Custom retreatment was statistically superior than standard retreatment for correction of lower and higher ocular aberrations.

Keywords: Astigmatism/surgery; Astigmatism/physiopathology; Myopia/surgery; Myopia/physiopathology; Keratomileusis, laser in situ/methods; Visual acuity/physiology; Retreatment 


\section{REFERÊNCIAS}

1. Urbano AP, Leal M, Urbano I. Descrição das aberrações oculares. In: Urbano AP, editor. Wavefront: cirurgia personalizada. Rio de Janeiro: Cultura Médica; 2006. p.101-13.

2. Chalita MR, Chavala S, Xu M, Krueger RR. Wavefront analysis in postLASIK eyes and its correlation with visual symptoms, refraction, and topography. Ophthalmology. 2004;111(3):447-53. Comment in: Ophthalmology. 2005; 112(6):1171; author reply 1171 .

3. Chalita MR, Krueger RR. Correlation of aberrations with visual acuity and symptoms. Ophthalmol Clin North Am. 2004;17(2):135-42, v-vi.

4. Urbano AP, Nosé R, Nosé W. Reoperações personalizadas. In: Alves MR, Chamon W, Nosé W, editores. Cirurgia refrativa. Rio de Janeiro: Cultura Médica; 2003. p.359-67.

5. Chalita MR, Chamon W, Schor P, Freda R, Krueger R. Resultados de literatura em tratamentos personalizados com excimer laser. In: Alves MR, Chamon W, Nosé W, editores. Cirurgia Refrativa. Rio de Janeiro: Cultura Médica; 2003. p.353-9.

6. Urbano AP. Cirurgias pimárias personalizadas. In: Urbano AP, editor. Wave front: cirurgia personalizada. Rio de Janeiro: Cultura Médica; 2006. p.375-95.

7. Huang D, Arif M. Spot size and quality of scanning laser correction of higher order wavefront aberrations. J Refract Surg. 2001;17(5):S588-91.

8. Martínez CE, Applegate RA, Klyce SD, McDonald MB, Medina JP, Howland HC. Effect of pupillary dilation on corneal optical aberrations after photorefractive keratectomy. Arch Ophthalmol. 1998;116(8):1053-62. Comment in: Arch Ophthalmol. 1998;116(8):1104-5.

9. Oshika T, Klyce SD, Applegate RA, Howland HC, El Danasoury MA. Comparison of corneal wavefront aberrations after photorefractive keratectomy and laser in situ keratomileusis. Am J Ophthalmol. 1999;127(1):1-7. Comment in: Am J Ophthalmol. 2006;141(6):1169; author reply 1169.

10. Seiler T, Kaemmerer M, Mierdel P, Krinke HE. Ocular optical aberrations after photorefractive keratectomy for myopia and myopic astigmatism. Arch Ophthalmol. 2000;118(1):17-21.

11. Moreno-Barriuso E, Lloves JM, Marcos S, Navarro R, Llorente L, Barbero S. Ocular aberrations before and after myopic corneal refractive surgery: LASIKinduced changes measured with laser ray tracing. Invest Ophthalmol Vis Sci. 2001;42(6):1396-403.

12. Marcos S, Barbero S, Llorente L, Merayo-Lloves J. Optical response to LASIK surgery for myopia from total and corneal aberration measurements. Invest Ophthalmol Vis Sci. 2001;42(13):3349-56.

13. Llorente L, Barbero S, Merayo J, Marcos S. Total and corneal optical aberrations induced by laser in situ keratomileusis for hyperopia. J Refract Surg. 2004;20(3):203-16.
14. Oliver KM, Hemenger RP, Corbett MC, O'Brart DP, Verma S, Marshall J, Tomlinson A. Corneal optical aberrations induced by photorefractive keratectomy. J Refract Surg. 1997;13(3):246-54.

15. Carones F, Vigo L, Scandola E. Wavefront-guided treatment of abnormal eyes using the LADARVision platform. J Refract Surg. 2003;19(6):S703-8.

16. Kim TI, Yang SJ, Tchah H. Bilateral comparison of wavefront-guided versus conventional laser in situ keratomileusis with Bausch and Lomb Zyoptix. J Refract Surg. 2004;20(5):432-8.

17. Awwad ST, El-Kateb M, Bowman RW, Cavanagh HD, McCulley JP. Wavefront-guided laser in situ keratomileusis with the Alcon CustomCornea and the VISX CustomVue: three-month results. J Refract Surg. 2004;20(5): S606-13

18. Williams DR, Yoon GY, Guirao A, Hofer H, Porter J. How far can we extend the limits of human vision. In: MacRae SM, Krueger RR, Applegate RA. Customized corneal ablation: the quest for super vision. Thorofare: Slack Incor porated; 2001. p.11-38.

19. Pettit GH, Campin JA, MacDonald MB, Krueger RR. Customized Ablation Using the Alcon CustomCornea Platform. In: Krueger RR, Applegate RA, Mac Rae SM. Wavefront customized visual correction: the quest for super vision II. Thorofare: Slack Incorporated; 2004. p.217-25.

20. Lawless MA, Hodge C, Rogers CM, Sutton GL. Laser in situ keratomileusis with Alcon CustomCornea. J Refract Surg. 2003;19(6):S691-6.

21. Kohnen T, Bühren J, Kühne C, Mirshahi A. Wavefront-guided LASIK with the Zyoptix 3.1 system for the correction of myopia and compound myopic astigmatism with 1-year follow-up: clinical outcome and change in higher order aberrations. Ophthalmology. 2004;111(12):2175-85.

22. Vongthongsri A, Phusitphoykai N, Naripthapan P. Comparison of wavefrontguided customized ablation vs. conventional ablation in laser in situ keratomileusis. J Refract Surg. 2002;18(3 Suppl):S332-5.

23. Mrochen M, Krueger RR, Bueeler M, Seiler T. Aberration-sensing and wavefront-guided laser in situ keratomileusis: management of decentered ablation. J Refract Surg. 2002;18(4):418-29.

24. Winkler von Mohrenfels C, Huber A, Gabler B, Herrmann W, Kempe A, Donitzky C, Lohmann CP. Wavefront-guided laser epithelial keratomileusis with the wavelight concept system 500. J Refract Surg. 2004;20(5):S565-9.

25. Castanera J, Serra A, Rios C. Wavefront-guided ablation with Bausch and Lomb Zyoptix for retreatments after laser in situ keratomileusis for myopia. J Refract Surg. 2004;20(5):439-43.

26. Chalita MR, Xu M, Krueger RR. Alcon CustomCornea wavefront-guided retreatments after laser in situ keratomileusis. J Refract Surg. 2004;20(5):S624-30.

27. Urbano AP, Nosé W. Comparação entre retratamento de LASIK personalizado e convencional. In: Urbano AP, editor. Wavefront: cirurgia personalizada. Rio de Janeiro: Cultura Médica; 2006. p.421-33. 